\title{
Differential Control of Band 3 Lateral and Rotational Mobility in Intact Red Cells
}

\author{
James D. Corbett, * Peter Agre, ${ }^{\ddagger}$ Jiri Palek, ${ }^{\mathbf{5}}$ and David E. Golan* \\ *Departments of Biological Chemistry and Molecular Pharmacology and of Medicine, Harvard Medical School; Division of \\ Hematology-Oncology, Brigham and Women’s Hospital, Boston, Massachusetts 02115; $\ddagger$ Departments of Medicine and of Biological \\ Chemistry, Johns Hopkins University School of Medicine, Baltimore, Maryland 21205; §Departments of Biomedical Research and of \\ Medicine, St. Elizabeth's Hospital, Boston, Massachusetts 02135
}

\begin{abstract}
Measurements of integral membrane protein lateral mobility and rotational mobility have been separately used to investigate dynamic protein-protein and protein-lipid interactions that underlie plasma membrane structure and function. In model bilayer membranes, the mobilities of reconstituted proteins depend on the size of the diffusing molecule and the viscosity of the lipid bilayer. There are no direct tests, however, of the relationship between mechanisms that control protein lateral mobility and rotational mobility in intact biological membranes. We have measured the lateral and rotational mobility of band 3 in spectrin-deficient red blood cells from patients with hereditary spherocytosis and hereditary pyropoikilocytosis. Our data suggest that band 3 lateral mobility is regulated by the spectrin content of the red cell membrane. In contrast, band 3 rotational mobility is unaffected by changes in spectrin content. Band 3 lateral mobility and rotational mobility must therefore be controlled by different molecular mechanisms. (J. Clin. Invest. 1994. 94:683-688.) Key words: erythrocyte membrane • hereditary spherocytosis • hereditary pyropoikilocytosis • fluorescence photobleaching recovery $\bullet$ polarized fluorescence depletion
\end{abstract}

\section{Introduction}

The fluid-mosaic model of membrane structure postulates that randomly distributed transmembrane proteins are free to diffuse within the membrane at rates determined by the viscosity of a homogeneous lipid milieu (1). This model has proved correct for model bilayer membranes, in which the mobilities of reconstituted proteins depend only on the size of the diffusing molecule and the viscosity of the lipid bilayer (2-5). In biological membranes, however, there appear to be additional constraints on the mobility and distribution of integral membrane proteins, including interactions with heterogeneously distributed mem-

Portions of this work were published in abstract form (1990. Blood. 76:8a).

Address correspondence to David E. Golan, M.D., Ph.D., Department of Biological Chemistry and Molecular Pharmacology, Harvard Medical School, 250 Longwood Avenue, Boston, MA 02115.

Received for publication 18 February 1994 and in revised form 13 April 1994.

J. Clin. Invest.

(c) The American Society for Clinical Investigation, Inc.

0021-9738/94/08/0683/06 \$2.00

Volume 94, August 1994, 683-688 brane lipids and with other integral or peripheral membrane proteins $(6,7)$.

The human red blood cell ( $\mathrm{RBC})^{1}$ membrane is a prototypical biological membrane composed of a lipid bilayer, in which integral membrane proteins are embedded, and a multicomponent protein skeleton that laminates the inner bilayer surface (8). Band 3, the RBC anion exchanger (9), is the major protein component of the RBC membrane (10) and the major site of attachment between the membrane skeleton and the bilayer (11). Spectrin, the major skeletal protein, is attached to the overlying bilayer through interactions involving ankyrin, which links spectrin to a portion $(10-15 \%)$ of band 3 , and protein 4.1 , which links spectrin to glycophorin $C(12,13)$ and possibly to glycophorin A $(14,15)$ and band 3 (16).

$40-70 \%$ of band 3 molecules are free to diffuse laterally in the normal intact RBC membrane. The mobile fraction of band 3 diffuses laterally at a rate of $1-2 \times 10^{-11} \mathrm{~cm}^{2} \mathrm{~s}^{-1}(17-$ 19). In contrast, RBC phospholipid analogues have fractional mobilities of $90-100 \%$ and translational diffusion coefficients of $1-5 \times 10^{-9} \mathrm{~cm}^{2} \mathrm{~s}^{-1}(17,20)$. Abundant evidence implicates the RBC membrane skeleton in controlling band 3 lateral mobility. Disruption of spectrin-ankyrin and ankyrin-band 3 linkages causes band 3 mobility to increase (21-24), as does treatment with proteases to remove the cytoplasmic domain of band $3(25,26)$ or treatment with ATP or 2,3-DPG (27) at levels that are sufficient to destabilize isolated RBC membrane skeletons (28). Oxidative crosslinking of spectrin induces slowing of band 3 diffusion $(17,29)$. Finally, band 3 is translationally immobile in Southeast Asian ovalocytosis (SAO) RBCs, in which band 3 forms linear stacks of oligomers in the plane of the membrane $(30)^{2}$ and structurally and functionally abnormal band 3 molecules bind more tightly than normal band 3 to ankyrin (18, $31-33)$. $^{2}$

Band 3 rotational mobility has been studied in normal RBC ghosts $(26,34-44)$, abnormal RBC ghosts $(45,46)$, and intact RBCs $(19,31,37){ }^{2}$ Rapidly rotating, slowly rotating, and rotationally immobile forms of band 3 appear to coexist in the membrane. At $37^{\circ} \mathrm{C}$ in normal RBC membranes, $20-25 \%$ of band 3 molecules rotate with correlation times $(\tau)<250 \mu \mathrm{s}$, $50-75 \%$ rotate with $\tau \sim 1-3 \mathrm{~ms}$, and 5-25\% are rotationally immobile on the time scale of the experiment $(19,37,40)$.

1. Abbreviations used in this paper: FPR, fluorescence photobleaching recovery; HPP, hereditary pyropoikilocytosis; HS, hereditary spherocytosis; KPBS, high potassium phosphate-buffered saline; PFD, polarized fluorescence depletion; RBC, red blood cell; SAO, Southeast Asian ovalocytosis.

2. Liu, S. C., J. Palek, S. J. Yi, P. E. Nichols, L. H. Derick, S. S. Chiou, D. Amato, J. D. Corbett, M. R. Cho, and D. E. Golan. Manuscript submitted for publication. 
About half of the rapidly rotating band 3 molecules may rotate with correlation times of $25-30 \mu \mathrm{s}$; this population could represent band 3 dimers rotating free of constraints other than the viscosity of the lipid bilayer (37). A number of experiments on RBC ghost membranes suggest that band 3 rotational mobility, like band 3 lateral mobility, is controlled by the RBC membrane skeleton $(26,36,37,43)$. In addition, SAO RBCs exhibit a very large fraction of rotationally immobile band $3(30,31,46) .^{2}$

Studies correlating defined molecular abnormalities in membrane skeletal proteins with band 3 mobility in intact RBCs from patients with hereditary hemolytic anemias provide unique opportunities to elucidate relationships among membrane skeletal interactions controlling band 3 lateral mobility and rotational mobility. In this study, we measure the lateral and rotational mobility of fluorescently labeled band 3 in matched samples of spectrin deficient RBCs from patients with hereditary spherocytosis (HS) and hereditary pyropoikilocytosis (HPP). We find that band 3 lateral diffusion rates are inversely proportional to the spectrin content of the RBC membrane. In contrast, band 3 rotational mobility is not affected by changes in spectrin content. Band 3 lateral mobility and rotational mobility must therefore be regulated by different molecular mechanisms.

\section{Methods}

Eosin maleimide was purchased from Molecular Probes, Inc. (Eugene, OR). Glucose oxidase and catalase were obtained from Sigma Chemical Co. (St. Louis, MO). Fresh blood was collected by venipuncture, anticoagulated with acid/citrate/dextrose, and shipped on ice to Boston, MA by overnight courier. Within $24 \mathrm{~h}$ of venipuncture the buffy coat was removed by aspiration and RBCs were washed 3 times and stored at $4^{\circ} \mathrm{C}$ in high potassium phosphate-buffered saline (KPBS) $(140 \mathrm{mM} \mathrm{KCl}$, $15 \mathrm{mM} \mathrm{NaPO}_{4}, 10 \mathrm{mM}$ glucose, $\mathrm{pH}$ 7.4). High potassium buffers were used to prevent possible cellular dehydration associated with deoxygenation and fluorescent labeling.

Biochemical analysis of $R B C$ membranes. The spectrin/band 3 ratio in HS, HS carrier, HPP, HPP carrier, and control RBC membranes was measured by densitometric scans of Coomassie blue-stained SDSpolyacrylamide gels, as described $(47,48)$. The range of spectrin/band 3 ratios for control RBC membranes was 0.95-1.05 (raw data), and experimental values were normalized to a control ratio of 1.00 . The spectrin content of HS, HS carrier, and control RBC membranes was also measured by spectrin radioimmunoassay, as described $(49,50)$.

Specific labeling of $R B C$ band $3.100 \mathrm{ml}$ of freshly washed packed RBCs were incubated with $40 \mathrm{ml}$ of eosin maleimide, $0.25 \mathrm{mg} / \mathrm{ml}$ in KPBS, at room temperature for $12 \mathrm{~min}$. Cells were then washed 3 times in KPBS with $1 \%$ BSA. Under these labeling conditions $>80 \%$ of the membrane-associated fluorescence was covalently bound to band 3 (51).

Lateral mobility measurements. Fluorescence photobleaching recovery (FPR) (52) was used to measure the lateral mobility of eosin labeled band 3 in membranes of intact RBCs. A Gaussian laser beam was focused to a spot on a labeled RBC in a fluorescence microscope. After a brief, intense photobleaching pulse, recovery of fluorescence was monitored by periodic low intensity pulses. Fluorescence recovery resulted from the lateral diffusion of unbleached fluorophores into the bleached area. Nonlinear least-squares analysis (53) of fluorescence recovery data yielded both the diffusion coefficient and the fraction of labeled band 3 molecules that were free to diffuse on the time scale of the experiment (17). Details of the electronics and optics used in our FPR apparatus have been published (19). All measurements were performed at $37^{\circ} \mathrm{C}$.

Rotational mobility measurements. Polarized fluorescence depletion (PFD) (54) was used to measure the rotational mobility of eosin labeled band 3 in membranes of intact RBCs. Eosin molecules were photoselected to the triplet state by an intense polarized laser pulse. The decay

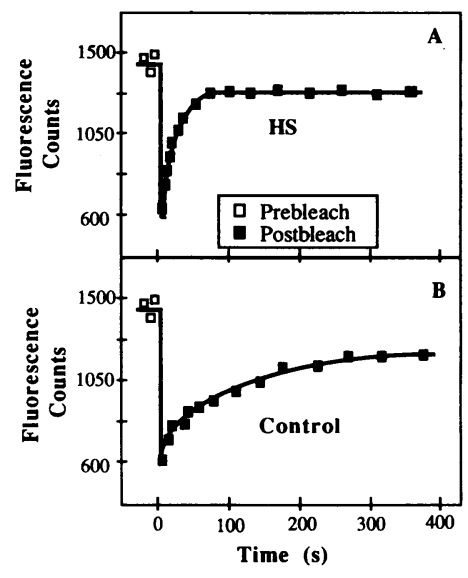

Fig. 1. Typical FPR curves depicting the lateral mobility of band 3 in intact RBCs from patients with HS $(A)$ and controls $(B)$. Normalized spectrin/band 3 ratios in the HS and control samples were 0.70 and 1.00 , respectively. Data points represent fluorescence counts at experimental times before and after the photobleaching pulse. Spectrin-deficient HS RBCs showed increased lateral diffusion coefficients relative to control RBCs, indicating increased freedom of band 3 lateral movement. HS, diffusion coefficient $=12 \times 10^{-11} \mathrm{~cm}^{2} \mathrm{~s}^{-1}$, fractional mobility $=78 \%$. Control, diffusion coefficient $=1.6 \times 10^{-11} \mathrm{~cm}^{2} \mathrm{~s}^{-1}$, fractional mobility $=70 \%$.

of induced anisotropy was measured by monitoring ground state fluorescence oriented parallel and perpendicular to the photoselection pulse $(37,54,55)$. Using our laser microscope photometer two exponential components of anisotropy decay and a residual anisotropy could be resolved, so data were fitted by nonlinear least squares analysis to the equation: $r(\mathrm{t})=r(\infty)+\alpha \cdot \exp \left(-\mathrm{t} / \tau_{1}\right)+\beta \cdot \exp \left(-\mathrm{t} / \tau_{2}\right)$, where $r(\mathrm{t})$ is the anisotropy at time $\mathrm{t}, r(\infty)$ is the residual anisotropy, and $\alpha$ and $\beta$ are the fractions of molecules with rotational correlation times $\tau_{1}$ and $\tau_{2}$, respectively. An enzyme oxygen scavenging system, consisting of 50 $\mathrm{U} / \mathrm{ml}$ glucose oxidase, $20 \mathrm{mM}$ glucose, and $10^{4} \mathrm{U} / \mathrm{ml}$ catalase, served to eliminate oxygen from the sample and thereby prevent quenching of the excited triplet state of eosin (55). Slides were prepared for PFD experiments by placing $3.2 \mu \mathrm{l}$ of a $10 \% \mathrm{RBC}$ suspension on a BSAcoated glass slide and using vacuum grease to seal a BSA-coated coverslip over the sample. Details of the electronics and optics used in our PFD apparatus have been published (19). All measurements were performed at $37^{\circ} \mathrm{C}$.

\section{Results}

We measured by FPR the lateral mobility of band 3 in intact RBCs from 8 patients (47) with severe, recessively inherited spherocytosis, 2 carriers of $\mathrm{HS}$, and 10 controls with normal RBC morphology. HS RBCs had spectrin contents by radioimmunoassay of $31-59 \%$ of control values and spectrin/band 3 ratios of $43-73 \%$ of control values $(47,49,50,56)$. Representative FPR curves for normal and spectrin deficient HS cells are shown in Fig. 1. When the logarithm of the band 3 diffusion coefficient was plotted against RBC spectrin content for HS, HS carrier, and control cells, an inverse linear relationship was observed with $R^{2}=0.81$ (Fig. $2 A$ ). Control diffusion coefficients were $0.7-2.4 \times 10^{-11} \mathrm{~cm}^{2} \mathrm{~s}^{-1}$, and the maximum observed diffusion coefficient was an order of magnitude greater. The extrapolated diffusion coefficient at $0 \%$ spectrin was 110 $\times 10^{-11} \mathrm{~cm}^{2} \mathrm{~s}^{-1}$, which is of the same magnitude as the band 3 "rapid diffusion limit" previously observed in RBC ghosts $(23,24,51)$. The results in RBCs from two HS carriers did not differ from those in RBCs from unrelated controls or from the results in RBCs from the splenectomized control. These data suggest that the rate of band 3 lateral diffusion is regulated primarily by RBC membrane spectrin content. Fractional mobilities of band 3 showed no significant correlation with RBC 


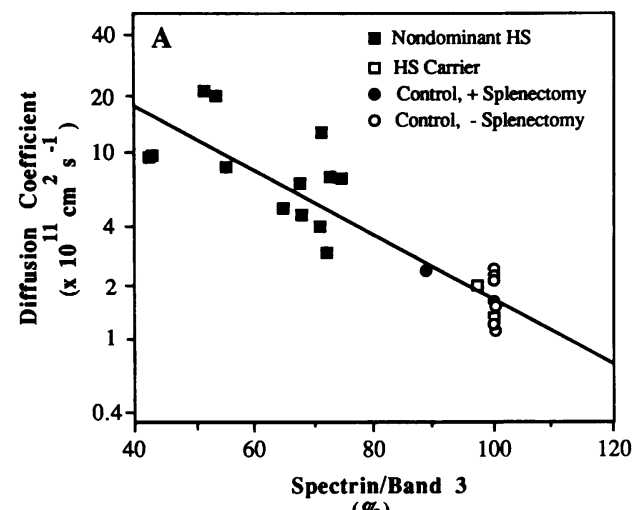

(\%)

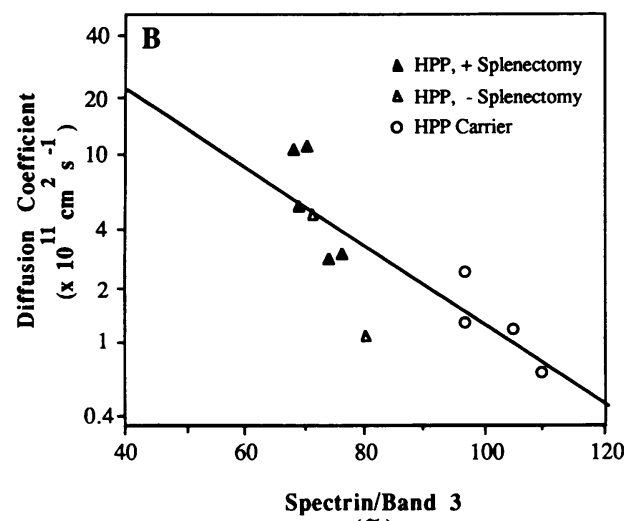

(\%)

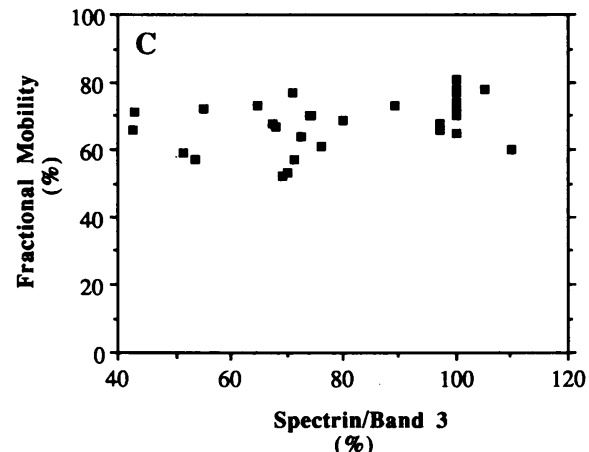

Fig. 2. Dependence of band 3 lateral-diffusion rate on normalized spectrin/band 3 ratio in intact RBCs from patients with HS $(A)$ and HPP $(B)$. Data points represent mean lateral diffusion coefficients of band 3 in membranes of 8-12 cells from one individual. The average SD for each data point was $48 \%$ (range, $24 \%-73 \%$ ). Filled symbols represent splenectomized individuals, open symbols indicate spleen retained. ( $A b$ scissa) Normalized ratio of spectrin to band 3 measured by densitometric scans of Coomassie blue stained SDS-polyacrylamide gels (47). (Ordinate) Band 3 lateral diffusion coefficient. Equations of best fit and correlation coefficients were: $\mathrm{HS}, \ln$ (diffusion coefficient) $=4.5$ $0.040 \cdot($ spectrin/band 3$), R^{2}=0.81 ; \mathrm{HPP}, \ln$ (diffusion coefficient) $=$ $5.1-0.049 \cdot($ spectrin/band 3$), R^{2}=0.69$. (C) Dependence of band 3 fractional mobility on normalized spectrin/band 3 ratio in intact $R B C s$ from patients with HS and HPP, carriers of HS and HPP, and controls. Data points represent mean fractional mobility of band 3 in membranes of 8-12 cells from one individual. The average SD for each data point was $18 \%$ (range, $9 \%-36 \%$ ). (Abscissa) Normalized ratio of spectrin to band 3 measured by densitometric scans of Coomassie blue stained SDS-polyacrylamide gels (47). (Ordinate) Band 3 fractional

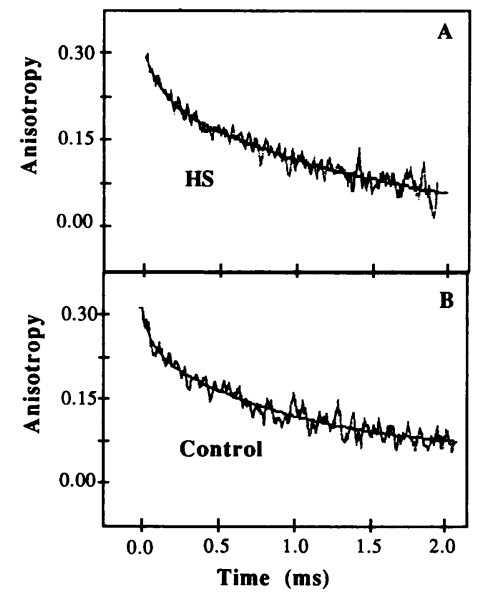

Fig. 3. Typical PFD curves showing the rotational mobility of band 3 in intact RBCs from patients with $\mathrm{HS}$ $(A)$ and controls $(B)$. Normalized spectrin/band 3 ratios in the HS and control samples were 0.68 and 1.00 , respectively. Data from $\sim 300$ cells were collected to obtain each curve. HS and control RBCs exhibited band 3 rotational mobilities that were not significantly different from one another. HS, $\alpha$ $=22 \%, \tau_{1}=147 \mu \mathrm{s}, \beta$

$=70 \%, \tau_{2}=1.9 \mathrm{~ms}, r(\infty)$

$=8 \%$. Control, $\alpha=27 \%, \tau_{1}$ $=71 \mu \mathrm{s}, \beta=58 \%, \tau_{2}=1.3$ $\mathrm{ms}, r(\infty)=15 \%$. spectrin content (Fig. $2 \widetilde{C}$ ). These data suggest that molecular interactions responsible for lateral immobilization of band 3, such as high-affinity binding of a fraction of band 3 to ankyrin (57), remain intact in HS RBCs.

We measured by PFD the rotational mobility of band 3 in intact RBCs from four of the patients with severe HS and two controls with normal RBC morphology. Control RBCs had 3 populations of band 3 molecules, including $\sim 25 \%$ rapidly rotating molecules $\left(\tau_{1}, \sim 100 \mu \mathrm{s}\right), \sim 60 \%$ slowly rotating molecules $\left(\tau_{2}, \sim 1.5 \mathrm{~ms}\right)$, and $\sim 15 \%$ rotationally immobile molecules. Spectrin-deficient HS RBCs had populations and correlation times of rapidly rotating, slowly rotating, and rotationally immobile band 3 molecules that were not significantly different from those in control RBCs (Fig. 3; Table I). These data indicate that specific binding interactions between band 3 and spectrin do not exist. If such interactions were present, a reduction in spectrin content would be expected to result in an increase in band 3 rotational mobility.

We also measured by FPR the lateral mobility of band 3 in intact RBCs from five patients with HPP and four carriers of HPP (48). HPP RBCs manifested moderate reductions in membrane spectrin content (spectrin/band 3 ratios, 68-80\% of control values). In addition, because the molecular defect in these HPP RBCs involved mutations in the spectrin $\alpha-1$ domain, the phenotype included weakened spectrin dimer-dimer association $(48,58-60)$. These RBCs exhibited band 3 lateral diffusion coefficients similar to those of HS and HS carrier RBCs with the same degree of spectrin deficiency. When the logarithm of the band 3 diffusion coefficient was plotted against spectrin content for HPP and HPP carrier cells, an inverse linear relationship was obtained with slope and rapid diffusion limit similar to those found for HS RBCs (Fig. $2 B$ ). Since HPP and HPP carrier RBCs defective in the spectrin dimer-dimer interaction manifested diffusion coefficients identical to those of HS and

mobility. The lateral diffusion coefficient of band 3 was inversely proportional to RBC spectrin content in HS and HPP RBCs, whereas the fraction of laterally mobile band 3 molecules did not depend on spectrin content. 
Table I. Rotational Mobility of Band 3 in HS and Control RBCs

\begin{tabular}{lccrrrr}
\hline \multicolumn{1}{c}{ Sample } & $\begin{array}{c}\text { Spectrin/Band } \\
\text { 3 ratio }\end{array}$ & $\alpha$ & $\tau_{1}$ & $\beta$ & $\tau_{2}$ & $\mathrm{r}(\infty)$ \\
\hline & & $\%$ & $\mu s$ & $\%$ & $m s$ & $\%$ \\
HS-1 & 0.54 & 25 & 89 & 70 & 2.2 & 5 \\
HS-2 & 0.68 & 22 & 147 & 70 & 1.9 & 8 \\
HS-3 & 0.72 & 26 & 50 & 61 & 1.2 & 13 \\
HS-4 & 0.74 & 28 & 44 & 50 & 1.2 & 22 \\
Control-1 & 1.00 & 18 & 50 & 63 & 1.4 & 8 \\
Control-2 & 1.00 & 27 & 71 & 58 & 1.3 & 15 \\
& & & & & & \\
\hline
\end{tabular}

PFD (21) was used to measure the rotational mobility of eosin labeled band 3 in intact RBCs from four patients with HS and two controls with normal RBC morphology. Experiments were performed at $37^{\circ} \mathrm{C}$. Rotational mobility parameters were determined by nonlinear leastsquares analysis. Chi-square values ranged from 0.17 to 0.42 for the equations corresponding to the best fit curves. $\alpha$ and $\beta$, fractions of band 3 molecules rotating with correlation times $\tau_{1}$ and $\tau_{2}$, respectively. $r(\infty)$, fraction of rotationally immobile band 3 molecules. Normalized spectrin/band 3 ratio was measured by densitometric scans of Coomassie blue-stained SDS-polyacrylamide gels (47).

HS carrier RBCs with the same degree of spectrin deficiency, the spectrin dimer-dimer interaction cannot be important for the regulation of band 3 lateral diffusion.

\section{Discussion}

In this work, measurements of band 3 mobility in membranes of intact RBCs from patients with two hereditary forms of hemolytic anemia are used to investigate hypotheses for the molecular interactions that control transmembrane protein lateral and rotational mobility. Band 3 lateral diffusion coefficients in HS and HPP RBCs are increased compared to those in control RBCs, and the degree of elevation in diffusion coefficient is inversely related to the RBC spectrin content. In contrast, band 3 rotational mobility in HS RBCs is identical to that in control RBCs. Finally, band 3 lateral mobility in HPP RBCs is similar to that in HS RBCs with the same degree of spectrin deficiency.

Several molecular mechanisms could underlie the dependence of band 3 lateral diffusion rate on $\mathrm{RBC}$ spectrin content. Spectrin, a flexible rod-like protein that may wind between junctional complexes at varying distances from the membrane bilayer (61), could act as a molecular "fence" to retard band 3 lateral movement. Spectrin could bind directly to the cytoplasmic domain of band 3 in a low affinity $\left(K_{\mathrm{d}} \sim 1-10 \mu \mathrm{M}\right)$ interaction, or it could modulate the affinity of a direct binding interaction between band 3 and ankyrin, protein 4.1, or another membrane skeletal component (51). Finally, interactions between spectrin and inner leaflet phospholipids (62) could alter the viscosity of the membrane lipid bilayer. The latter mechanism is unlikely, because we found no significant difference between the lateral diffusion coefficient or fractional mobility of fluorescein phosphatidylethanolamine, a fluorescent phospholipid analogue, in HS and control RBCs (data not shown).

Measurements of band 3 rotational mobility in HS and control RBCs suggest that mechanisms involving steric hindrance to band 3 lateral diffusion are more likely than mechanisms involving direct binding interactions. Lateral mobility measure- ments provide information about long-range dynamic interactions ( $\mu \mathrm{m}$ scale) between the diffusing species and membrane protein or lipid components, whereas rotational mobility measurements yield information concerning short-range dynamic interactions (nm scale). Thus, interactions that regulate membrane protein lateral mobility include specific binding, steric hindrance, self-aggregation, heterocomplex formation, and domain formation $(6,63)$, while only direct binding interactions and protein oligomerization appear to be important in controlling rotational mobility (64). Since the rotational mobility of band 3 in spectrin-deficient HS RBCs is identical to that in control RBCs, the average oligomerization state of band 3 cannot be a function of RBC spectrin content, and significant binding interactions between band 3 and spectrin cannot exist. Because spectrin-band 3 interactions are critical in regulating the lateral but not the rotational diffusion of band 3 in intact RBCs, our data favor the steric hindrance (fence) model of lateral mobility regulation (65) over the direct binding model (reviewed in reference 51). This conclusion is supported indirectly by experiments on band 3 mobility in RBC ghost membranes $(22,25,26,35,66)$.

The molecular interactions that control band 3 rotational diffusion appear to involve both band 3 self-association (aggregation) in the plane of the $R B C$ membrane $(36,41)$ and specific binding interactions between the cytoplasmic domain of band 3 and membrane skeletal proteins $(4,5,37,67)$ such as ankyrin. In spectrin-deficient HS RBCs, ankyrin binding sites on spectrin may remain in sufficient numbers to provide restriction of band 3 rotational mobility through band 3-ankyrin complex formation. Band 3 interactions with proteins $4.1(16)$ or $4.2(68,69)$, which would not be perturbed in spectrin-deficient RBCs, could also be responsible for rotational mobility restriction in HS RBCs (67).

One version of the steric hindrance model for regulation of band 3 lateral diffusion rates involves the possibility of dynamic interconversion between spectrin dimers (open fences) and tetramers (closed fences) at the inner surface of the RBC membrane (70). HS and HPP RBCs share the spectrin-deficient phenotype. The spectrin dimer-dimer interaction is functionally normal in HS RBCs and weakened in HPP RBCs, however. A comparison between band 3 lateral diffusion rates in intact HS and HPP RBCs should therefore provide a test of the importance of the spectrin dimer $\leftrightarrow$ tetramer interconversion in controlling band 3 lateral mobility. We find that the lateral mobility of band 3 in HPP and HPP carrier RBCs is similar to that of HS and HS carrier RBCs with the same degree of spectrin deficiency. These data suggest that control of band 3 lateral mobility depends on the absolute number of spectrin molecules in the membrane skeleton and not on the state of spectrin oligomerization. Thus, the spectrin dimer-dimer interaction is unlikely to be important for the regulation of band 3 lateral diffusion. Steric hindrance interactions between spectrin and band 3 must therefore be mediated not by alterations in the spectrin oligomerization state but rather by other dynamic changes in spectrin structure, such as thermodynamic fluctuations of spectrin dimers toward (closed fence) or away from (open fence) the inner leaflet of the RBC membrane.

In conclusion, these data suggest that band 3 lateral mobility and rotational mobility are controlled by different molecular interactions. The spectrin-based membrane skeleton appears to be critical in regulating the lateral $(22,66)$ but not the rotational $(25,26,35)$ diffusion of band 3 . This deduction favors the steric 
hindrance model of lateral mobility regulation (65) over the specific binding site model (reviewed in reference 51). In contrast, molecular binding interactions involving the cytoplasmic domain of band 3 , including increased affinity for ankyrin (31, $46),{ }^{2}$ increased binding of abnormal or denatured hemoglobin (19) or glycolytic enzymes (37), and possibly oxidative crosslinking of band 3 to large aggregates, are likely to be required for rotational immobilization of this protein. Future studies will define further the roles of membrane skeletal proteins that bind directly to the cytoplasmic domain of band 3, such as ankyrin and protein 4.2 , in regulating the distribution of band 3 molecules in intact $\mathrm{RBCs}$ among rapidly rotating, slowly rotating, and rotationally immobile populations.

\section{Acknowledgments}

We are grateful to the patients and controls for generous donations of blood samples. We thank Adam Abroms, Carl Brown, Yung Han, and Patrick Yacono for outstanding technical assistance. We thank Alan Kleinfeld for the nonlinear least-squares algorithm used to analyze polarized fluorescence depletion data.

This work was supported by National Institutes of Health grants HL-32854 (D.E.Golan), HL-15157 (D.E.Golan and J.Palek), HL-27215 and HL-37462 (J.Palek), and HL-33991 (P.Agre). J.D.Corbett was supported by National Institutes of Health training grant HL-07623 for a portion of this work.

\section{References}

1. Singer, S. J., and G. L. Nicolson. 1972. The fluid mosaic model of the structure of cell membranes. Science (Wash. DC). 175:720-731.

2. Saffman, P. G., and M. Delbruck. 1975. Brownian motion in biological membranes. Proc. Natl. Acad. Sci. USA. 72:3111-3113.

3. Vaz, W. L. C., F. Goodsaid-Zalduondo, and K. Jacobson. 1984. Lateral diffusion of lipids and proteins in bilayer membranes. FEBS (Fed. Eur. Biochem. Soc.) Lett. 174:199-204.

4. Muhlebach, T., and R. J. Cherry. 1985. Rotational diffusion and selfassociation of band 3 in reconstituted lipid vescles. Biochemistry. 24:975-983.

5. Morrison, J. E. G., T. J. Muhlebach, and R. J. Cherry. 1986. Rotational diffusion of band 3 reconstituted into lipid vesicles: An application of global analysis. Biochem. Soc. Trans. 14:885-886.

6. Nicolson, G. L. 1976. Transmembrane control of the receptors on normal and tumor cells. I. Cytoplasmic influence over surface components. Biochim. Biophys. Acta. 457:57-108.

7. Jacobson, K., A. Ishihara, and R. Inman. 1987. Lateral diffusion of proteins in membranes. Annu. Rev. Physiol. 49:163-175.

8. Bennett, V. 1985. The membrane skeleton of human erythrocytes and its implications for more complex cells. Annu. Rev. Biochem. 54:273-304.

9. Cabantchik, Z. I., P. A. Knauf, and A. Rothstein. 1978. The anion transport system of the red blood cell: the role of membrane protein evaluated by the use of 'probes'. Biochim. Biophys. Acta. 515:239-302.

10. Steck, T. L. 1974. The organization of proteins in the human red blood cell membrane. J. Cell Biol. 62:1-19.

11. Low, P. S. 1986. Structure and function of the cytoplasmic domain of band 3: Center of erythrocyte membrane-peripheral protein interactions. Biochim. Biophys. Acta. 864:145-167.

12. Alloisio, N., L. Morle, D. Bachir, D. Guetarni, P. Colonna, and J. Delaunay. 1985. Red cell membrane sialoglycoprotein $\beta$ in homozygous and heterozygous 4.1(-) hereditary elliptocytosis. Biochim. Biophys. Acta. 816:57-62.

13. Reid, M. E., J. A. Chasis, and N. Mohandas. 1987. Identification of a functional role for human erythrocyte sialoglycoproteins $\beta$ and $\gamma$. Blood. 69:1068-1072.

14. Anderson, R. A., and R. E. Lovrien. 1984. Glycophorin is linked by band 4.1 protein to the human erythrocyte membrane skeleton. Nature (Lond.). 307:655-658.

15. Anderson, R. A., and V. T. Marchesi. 1985. Regulation of the association of membrane skeletal protein 4.1 with glycophorin by a polyphosphoinositide. Nature (Lond.). 318:295-299.

16. Pasternack, G. R., R. A. Anderson, T. L. Leto, and V. T. Marchesi. 1985 Interactions between protein 4.1 and band 3. J. Biol. Chem. 260:3676-3683.

17. Golan, D. E., C. S. Brown, C. M. L. Cianci, S. T. Furlong, and J. P. Caulfield. 1986. Schistosomula of Schistosoma mansoni use lysophosphatidylcho- line to lyse adherent red blood cells and immobilize red cell membrane components. J. Cell Biol. 103:819-828.

18. Liu, S. C., S. Zhai, J. Palek, D. E. Golan, D. Amato, K. Hassan, G. T. Nurse, D. Babona, T. Coetzer, P. Jarolim, M. Zaik, and S. Borwein. 1990. Molecular defect of the band 3 protein in Southeast Asian ovalocytosis. N. Engl. J. Med. 323:1530-1538

19. Corbett, J. D., and D. E. Golan. 1993. Band 3 and glycophorin are progressively aggregated in density-fractionated sickle and normal red blood cells. $J$. Clin. Invest. 91:208-217.

20. Golan, D. E., M. R. Alecio, W. R. Veatch, and R. R. Rando. 1984. Lateral mobility of phospholipid and cholesterol in the human erythrocyte membrane: Effects of protein-lipid interactions. Biochemistry. 23:332-339.

21. Fowler, V., and V. Bennett. 1978. Association of spectrin with its membrane attachment site restricts lateral mobility of human erythrocyte integral membrane proteins. J. Supramol. Struct. 8:215-221.

22. Golan, D. E., and W. Veatch. 1980. Lateral mobility of band 3 in the human erythrocyte membrane studied by fluorescence photobleaching recovery: evidence for control by cytoskeletal interactions. Proc. Natl. Acad. Sci. USA. 77:2537-2541.

23. Golan, D. E. 1982. Dynamic interactions in the human erythrocyte mem brane: Studies on the lateral mobility of band 3, phospholipid, and cholesterol. Yale University, Ph.D. thesis.

24. Golan, D. E., and W. Veatch. 1982. Lateral mobility of band 3 in the human erythrocyte membrane. Control by ankyrin-mediated interactions. Biophys. J. 37:177a. (Abstr.)

25. Tsuji, A., and S. Ohnishi. 1986. Restriction of the lateral motion of band 3 in the erythrocyte membrane by the cytoskeletal network: Dependence on spectrin association state. Biochemistry. 25:6133-6138.

26. Tsuji, A., K. Kawasaki, and S. Ohnishi. 1988. Regulation of band 3 mobilities in erythrocyte ghost membranes by protein association and cytoskeletal meshwork. Biochemistry. 27:7447-7452.

27. Schindler, M., D. E. Koppel, and M. P. Sheetz. 1980. Modulation of membrane protein lateral mobility by polyphosphates and polyamines. Proc. Natl. Acad. Sci. USA. 77:1457-1461.

28. Sheetz, M. P., and J. Casaly. 1980. 2,3-Diphosphoglycerate and ATP dissociate erythrocyte membrane skeletons. J. Biol. Chem. 255:9955-9960.

29. Smith, D. K., and J. Palek. 1982. Modulation of lateral mobility of band 3 in the red cell membrane by oxidative cross-linking of spectrin. Nature (Lond.). 297:424-425.

30. Che, A., R. J. Cherry, L. H. Bannister, and A. R. Dluzewski. 1993. Aggregation of band 3 in hereditary ovalocytic red blood cell membranes: Electron microscopy and protein rotational diffusion studies. J. Cell Sci. 105:655-660.

31. Liu, S. C., S. J. Yi, L. H. Derick, P. E. Nichols, D. Amato, J. D. Corbett, D. E. Golan, and J. Palek. 1993. Characterization of the band 3 protein in Southeast Asian ovalocytosis (SAO). Interrelationships among band 3 self-association, ankyrin binding, rotational and lateral mobilities. Clin. Res. 41:135a. (Abstr.)

32. Mohandas, N., R. Winardi, D. Knowles, A. Leung, M. Parra, E. George, J. Conboy, and J. Chasis. 1992. Molecular basis for membrane rigidity of hereditary ovalocytosis. J. Clin. Invest. 89:686-692.

33. Schofield, A. E., D. M. Reardon, and M. J. A. Tanner. 1992. Defective anion transport activity of the abnormal band 3 in hereditary ovalocytic red blood cells. Nature (Lond.). 355:836-838.

34. Austin, R. H., S. S. Chan, and T. M. Jovin. 1979. Rotational diffusion of cell surface components by time-resolved phosphorescence anisotropy. Proc. Natl. Acad. Sci. USA. 76:5650-5654.

35. Cherry, R. J., A. Burkli, M. Busslinger, G. Schneider, and G. R. Parish. 1976. Rotational diffusion of band 3 proteins in the human erythrocyte membrane. Nature (Lond.). 263:389-393.

36. Clague, M. J., J. P. Harrison, and R. J. Cherry. 1989. Cytoskeletal restraints of band 3 rotational mobility in human erythrocyte membranes. Biochim. Biophys. Acta. 981:43-50.

37. Matayoshi, E. D., and T. M. Jovin. 1991. Rotational diffusion of band 3 in erythrocyte membranes. 1. Comparison of ghosts and intact cells. Biochemistry. 30:3525-3538.

38. Muhlebach, T., and R. J. Cherry. 1982. Influence of cholesterol on the rotation and self-association of band 3 in the human erythrocyte membrane Biochemistry. 21:4225-4228.

39. Nigg, E. A., C. Bron, M. Girardet, and R. J. Cherry. 1980. Band 3Glycophorin A association in erythrocyte membranes demonstrated by combining protein diffusion measurements with antibody-induced cross-linking. Biochemistry. 19:1887-1893.

40. Nigg, E. A., and R. J. Cherry. 1979. Influence of temperature and cholesterol on the rotational diffusion of band 3 in the human erythrocyte membrane. Biochemistry. 18:3457-3465.

41. Nigg, E. A., and R. J. Cherry. 1979. Dimeric association of band 3 in the erythrocyte membrane demonstrated by protein diffusion measurements. Nature (Lond.). 277:493-494.

42. Nigg, E. A., M. Kessler, and R. J. Cherry. 1979. Labeling of human erythrocyte membranes with eosin probes used for protein diffusion measure- 
ments: Inhibition of anion transport and photooxidative inactivation of acetylcholinesterase. Biochim. Biophys. Acta. 550:328-340.

43. Nigg, E. A., and R. J. Cherry. 1980. Anchorage of a band 3 population at the erythrocyte cytoplasmic membrane surface: Protein rotational diffusion measurements. Proc. Natl. Acad. Sci. USA. 77:4702-4706.

44. Nigg, E. A., C. G. Gahmberg, and R. J. Cherry. 1980. Rotational diffusion of band 3 proteins in membranes from $\operatorname{En}(\mathrm{a}-)$ and neuraminidase-treated normal human erythrocytes. Biochim. Biophys. Acta. 600:636-642.

45. Tilley, L., M. Foley, R. F. Anders, A. R. Dluzewski, W. B. Gratzer, G. L. Jones, and W. H. Sawyer. 1990. Rotational dynamics of the integral membrane protein, band 3, as a probe of the membrane events associated with Plasmodium falciparum infections of human erythrocytes. Biochim. Biophys. Acta. 1025:135142.

46. Tilley, L., G. B. Nash, G. L. Jones, and W. H. Sawyer. 1991. Decreased rotational diffusion of band 3 in melanesian ovalocytes from Papua, New Guinea. J. Membr. Biol. 121:59-66.

47. Agre, P., E. P. Orringer, and V. Bennett. 1982. Deficient red-cell spectrin in severe, recessively inherited spherocytosis. N. Engl. J. Med. 306:1155-1161.

48. Coetzer, T. L., and J. Palek. 1986. Partial spectrin deficiency in hereditary pyropoikilocytosis. Blood. 67:919-924.

49. Agre, P., J. F. Casella, W. H. Zinkham, C. McMillan, and V. Bennett. 1985. Partial deficiency of erythrocyte spectrin in hereditary spherocytosis. Nature (Lond.). 314:380-383.

50. Agre, P., A. Asimos, J. F. Casella, and C. McMillan. 1986. Inheritance pattern and clinical response to splenectomy as a reflection of erythrocyte spectrin deficiency in hereditary spherocytosis. N. Engl. J. Med. 315:1579-1583.

51. Golan, D. E. 1989. Red blood cell membrane protein and lipid diffusion. In Red Blood Cell Membranes. P. Agre and J. C. Parker, editors. Marcel Dekker, New York. 367-400.

52. Axelrod, D., D. E. Koppel, J. Schlessinger, E. Elson, and W. W. Webb. 1976. Mobility measurements by analysis of fluorescence photobleaching recovery kinetics. Biophys. J. 16:1055-1069.

53. Bevington, P. R. 1969. Data reduction and error analysis for the physical sciences. McGraw-Hill Inc., New York. 336 pp.

54. Yoshida, T. M., and B. G. Barisas. 1986. Protein rotational motion in solution measured by polarized fluorescence depletion. Biophys. J. 50:41-53.

55. Johnson, P., and P. B. Garland. 1981. Depolarization of fluorescence depletion. FEBS (Fed. Eur. Biochem. Soc.) Lett. 132:252-256.

56. Costa, F. F., P. Agre, P. C. Watkins, J. C. Winkelmann, T. K. Tang, K. M. John, S. E. Lux, and B. G. Forget. 1990. Linkage of dominant hereditary spherocytosis to the gene for the erythrocyte membrane-skeleton protein ankyrin. N. Engl. J. Med. 323:1046-1050.
57. Bennett, V. 1982. The molecular basis for membrane-cytoskeleton association in human erythrocytes. J. Cell. Biochem. 18:49-65.

58. Palek, J., S. Liu, P. Liu, J. Prchal, and R. P. Castleberry. 1981. Altered assembly of spectrin in red cell membranes in hereditary pyropoikilocytosis. Blood. 57:130-139.

59. Liu, S. C., L. H. Derick, P. Agre, and J. Palek. 1990. Alteration of the erythrocyte membrane skeletal ultrastructure in hereditary spherocytosis, hereditary elliptocytosis, and pyropoikilocytosis. Blood. 76:198-205.

60. Coetzer, T., J. Palek, J. Lawler, S. C. Liu, P. Jarolim, M. Lahav, J. T. Prchal, W. Wang, B. P. Alter, G. Schewitz, V. Mankad, R. Gallanello, and A. Cao. 1990. Structural and functional heterogeneity of a spectrin mutations involving the spectrin heterodimer self-association site: relationships to hematologic expression of homozygous hereditary elliptocytosis and hereditary pyropoikilocytosis. Blood. 75:2235-2244.

61. Shotton, D. M., B. E. Burke, and D. Branton. 1979. The molecular structure of human erythrocyte spectrin: biophysical and electron microscopic studies. $J$. Mol. Biol. 131:303-329.

62. Bitbol, M., C. Dempsey, A. Watts, and P. F. Devaux. 1989. Weak interaction of spectrin with phosphatidylcholine-phosphatidylserine multilayers: $A{ }^{2} \mathbf{H}$ and ${ }^{31}$ P NMR study. FEBS (Fed. Eur. Biochem. Soc.) Lett. 244:217-222.

63. Elson, E. L. 1986. Membrane dynamics studied by fluorescence correlation spectroscopy and photobleaching recovery. Soc. Gen. Physiol. Ser. 40:367-383.

64. Cherry, R. J. 1979. Rotational and lateral diffusion of membrane proteins. Biochim. Biophys. Acta. 599:289-327.

65. Sheetz, M. P. 1983. Membrane skeletal dynamics: Role in modulation of red cell deformability, mobility of transmembrane proteins, and shape. Semin. Hematol. 20:175-188.

66. Sheetz, M. P., M. Schindler, and D. E. Koppel. 1980. Lateral mobility of integral membrane proteins is increased in spherocytic erythrocytes. Nature (Lond.). 285:510-512.

67. Wyatt, K., and R. J. Cherry. 1992. Both ankyrin and band 4.1 are required to restrict the rotational mobility of band 3 in the human erythrocyte membrane. Biochim. Biophys. Acta. 1103:327-330.

68. Korsgren, C., and C. M. Cohen. 1986. Purification and properties of human erythrocyte band 4.2: Association with the cytoplasmic domain of band 3. J. Biol. Chem. 261:5536-5543.

69. Korsgren, C., and C. M. Cohen. 1988. Associations of human erythrocyte band 4.2. Binding to ankyrin and to the cytoplasmic domain of band 3. J. Biol. Chem. 263:10212-10218.

70. Koppel, D. E., M. P. Sheetz, and M. Schindler. 1981. Matrix control of protein diffusion in biological membranes. Proc. Natl. Acad. Sci. USA. 78:35763580 . 